\title{
Configuration Software Design for Wireless Ad Hoc Network Monitoring System in Intelligent Building
}

\author{
Xiuchong Gao*, Guiqing Zhang, Chenlu Tian and Yong Li \\ School of Information \& Electrical Engineering, Shandong Jianzhu University, Jinan, P, R. China \\ ${ }^{*}$ Corresponding author
}

\begin{abstract}
In the field of intelligent building research, in order to meet the business needs of developing intelligent device monitoring software flexibly and quickly, a configuration software including configuration module and running module is designed for the wireless ad hoc network monitoring system in intelligent building. In this thesis, the overall architecture of the configuration software is given, and the components such as intelligent device model library, data processing algorithm model library and other components are designed. The configuration software can be used to develop the monitoring software of intelligent devices with space as a management unit. Especially when the number or type of intelligent devices changes, users can rapidly complete the reconstruction of the monitoring software by only configuring the intelligent devices model corresponding to the intelligent devices which have been changed. At present, A monitoring software based on the configuration software has been verified and applied in the wireless ad hoc network monitoring system of Shandong Jianzhu University Passive Experimental Building, and the experimental results show that the design of the configuration software is feasible and effective.
\end{abstract}

Keywords-intelligent building; wireless ad hoc network monitoring system; intelligent device model library; data processing algorithm model library; monitoring software; configuration software

\section{INTRODUCTION}

With the continuous development of computer technology, there have been some limitations in the current architecture of building intelligent system, such as large configuration workload, lacks of scalability and flexibility, and the difficulties in realizing coordinated operation function cross systems $^{[1]}$. In [2], a architecture of building intelligent system is proposed, which can realize self organization, plug-and-play of intelligent devices, and flat systems. The application of the wireless ad hoc network monitoring system under the architecture can realize the coordinated operation of the intelligent device in the same space, and the development of the monitoring software for intelligent devices is the key to realize the intelligentization of the building. At present ,the monitoring software of intelligent devices is generally developed for specific engineering requirements, but when the intelligent device changes, the software usually needs to be reprogrammed, in which there is a great shortage of flexibility and extensibility. The emergence of configuration software provides a new way to solve the problems above. Users can configure their own projects, and quickly build the monitoring software that can meets their needs, so as to complete the monitoring task ${ }^{[3]}$.
This paper introduces a kind of configuration software for the wireless ad-network monitoring system in intelligent building, which is applied to construct the intelligent device monitoring software. Through the simple drag and drop, the monitoring software can be constructed and reconstructed quickly and flexibly by using intelligent device model and data processing algorithm model without tedious coding.

\section{COMPOSITION OF THE WIRELESS AD HOC NETWORK MONITORING SYSTEM IN INTELLIGENT BUILDING}

The architecture of the wireless ad hoc network monitoring system in intelligent building is shown in Figure 1.there are several intelligent devices that communicate with each other through the wireless ad hoc network in the room(space unit) ,such as IoT node, curtains, lamps, sockets, sensors, and so on. Specifically, IoT node is a kind of intelligent node with the data preprocessing and device management function[4]. The intelligent devices can realize ad hoc network and Plugand-Play when they are increased or decreased from the wireless ad hoc monitoring system, and meanwhile the data information of the related intelligent devices will be automatically added into or deleted from the data frame that will be uploaded to the server through the IoT node.

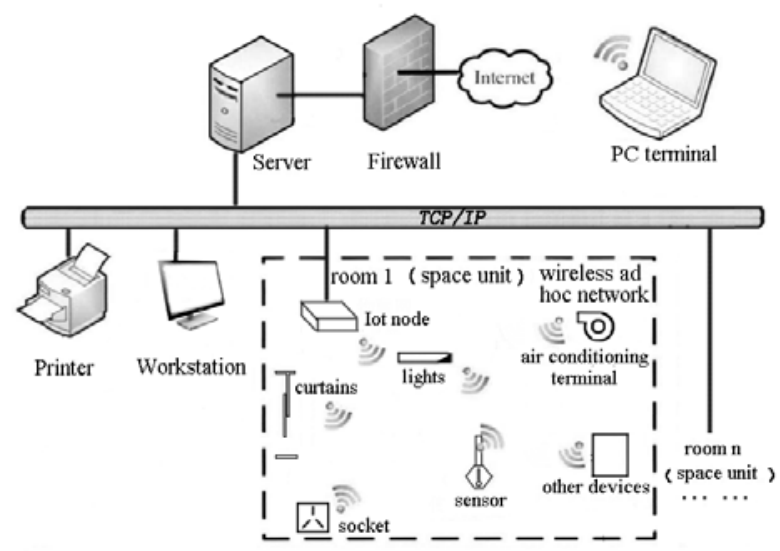

FIGURE I. THE ARCHITECTURE OF THE WIRELESS AD HOC MONITORING SYSTEM IN INTELLIGENT BUILDING

\section{CONFIGURATION SOFTWARE DESIGN}

\section{A. Architecture Design}

As shown in Figure 2, the configuration software for the wireless ad hoc network monitoring system in intelligent 
building is consisted by the configuration module and the running module:

- Configuration module: the component for constructing monitoring software, is used to realize the configuration of the monitoring interface and the data points, and saves the the project by outputting the data file in a specific format. It includes configuration component, intelligent device model library and data processing algorithm model library.

- Running module: the component for running monitoring software is used to load the data file which is output from the configuration component, realizes the visualization of the intelligent device operation data, and control the intelligent device through the communication component. It includes running component, data center (including real-time database and historical database) as well as the componentbased communication Interface (communication component).The historical database adopts the mainstream relational database, such as Oracle or SQL Server.

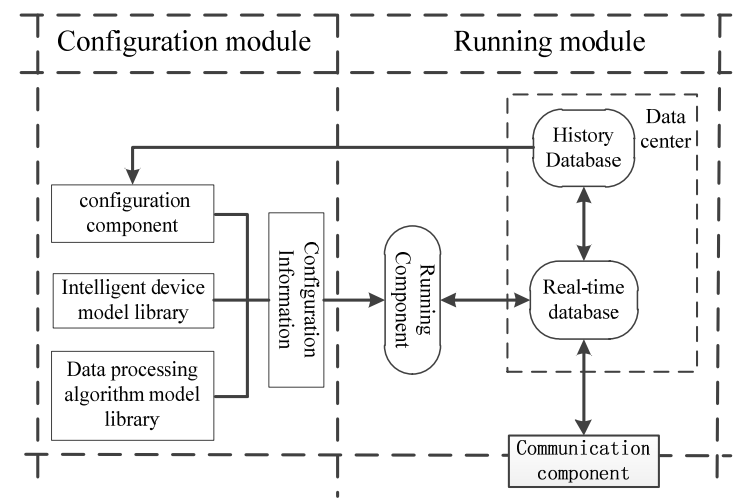

FIGURE II. CONFIGURATION SOFTWARE ARCHITECTURE DIAGRAM

\section{B. Components Design}

The configuration software is composed of the following core components: configuration component, intelligent device model library, data processing algorithm model library, data center, running component and communication component. The design and functions of each component are as follows:

- Data Center: It is the center of the whole configuration software and defines the full set of intelligent equipment's' data type. There are two parts, real-time database and historical database, in the data center that the first one is constructed in a memory mapping file based way, while the other one back the data of realtime database up in the regular intervals.

- Configuration component: it is used to customize the monitoring interface, configure the connection between the operating parameters of the intelligent device model and the data center data point, and save configuration engineering by exporting a specific format of data files. It contains the monitoring interface designer, the parameter configuration tool, the control logic editor, and the engineering file output tool.

- Intelligent device model library: providing common intelligent device models, the properties of which include devices model's types, operating parameters, operation interfaces, and the logos. The intelligent device model library is provided in the form of dynamic link library, and can be expend by software developers according to the actual needs.

- Data processing model library: providing common data processing models, the properties of which include types, number of input, and number of output, parameters, and logos. The data processing model library is provided in the form of dynamic link library, and can be expend by software developers according to the actual needs.

- Running component: it is responsible for loading and running the data files output from the configuration module, interact with the data center based on the C/S architecture, provide the man-machine interaction interface, implement the data visualization and remote manipulation of intelligent devices.

- Communication component: it is responsible for the communication between real-time database and the Iot nodes, mainly the encapsulation of TCP/IP communication protocol. Protocol data frame information includes the number, type and operation data of intelligent devices that in space unit. Communication component is provided for real-time database calls in dynamic link libraries.

\section{Interfaces Design}

1) Interaction between the configuration component and the data center: The interaction between the configuration component and the data center is based on the WebService interface, and configuration component mainly interacts with the historical database of the data center.According to the user's different needs, the configuration component and data center can be centralized deployment or decentralized deployment based on $\mathrm{C} / \mathrm{S}$ architecture. When users start to configure monitoring software based on the configuration component,they need to login the authorized account firstly,and establish the communication link with the data center. The main process of interaction is as follows:

The configuration component sends a data frame to the data center,which includes user's ID and password, and the data center judges whether the user is a legitimate user according to the user's ID and password provided by the user;

If the user is legitimate, data center will package the ID, name, type, and operating parameters of all the smart devices under the user right to the configuration component. If not, a denial of service message will be returned.

2) Interaction between the running component and the data center: The interaction between the running component and the data center is based on the WCF interface, and the running component mainly interacts with the real-time 
database. Similar to the configuration component, the running component and data center can be centralized deployment or decentralized deployment based on $\mathrm{C} / \mathrm{S}$ architecture. the main process is as follows:

According to the configuration information in the data file, the running component will send requests to the data center for the real-time operating data of the intelligent device or send the control instruction, the data center returns the corresponding data or the result according to the request, and then the running component refreshes monitoring interface with the data or the result.

When the real-time operating data of intelligent device which is received by the data center throgh the communication component changes, the data that is changed will be pushed to the running component immediately.

When the running component is closed, the logout instruction is sent to the data center and the communication link is also disconnected. After receiving the logout instruction, the data center stops the service of the running component.

\section{Design of the Intelligent Device Model Library}

The intelligent device model in traditional configuration software, including model logo and operating parameters, is not professional enough for building industry, which has led to a great limitation on building friendly monitoring interface and data collecting, storing and analyzing of intelligent device. Based on the object-oriented theory, fourteen intelligent device models are defined according to the actual requirements. The model information includes the name, type, operation parameters, operation interface and logo of the device.

The intelligent device model is classified and established according to the common intelligent device in the intelligent building. Based on the general electrical equipment class, detection sensor class, general class three types as the principle of classification, the intelligent devices are divided into 14 categories which include switch, socket, curtain controllers, airconditioning terminal, infrared forwarding module, temperature/humidity/light intensity, PM2.5/10, Person detection, $\mathrm{CO} 2$ concentration, $\mathrm{CO}$ concentration, electrical parameter detection, Abnormal alarm, Home mode controller and general intelligent device model. The intelligent device model library is not limited to the fourteen models, and the software developers can expand the custom model according to the actual requirements. The typical intelligent device model design in the fourteen models is shown in Table 1, and the logo of the typical model is shown in Figure 3.

TABLE I. TYPICAL INTELLIGENT DEVICE MODEL

\begin{tabular}{|l|l|l|l|}
\hline \multicolumn{1}{|c|}{ Name } & \multicolumn{1}{|c|}{ Type } & \multicolumn{1}{|c|}{ Operating parameters } & \multicolumn{1}{c|}{$\begin{array}{c}\text { Operation } \\
\text { interface }\end{array}$} \\
\hline Socket & Common & State & ON;OFF \\
\hline Switch & $\begin{array}{l}\text { Single; } \\
\text { Double; } \\
\text { Triple }\end{array}$ & State & ON;OFF \\
\hline $\begin{array}{l}\text { Temperature } \\
\text { Humidity } \\
\text { Lightintensity }\end{array}$ & Common & $\begin{array}{l}\text { Temperature value; } \\
\text { Humidity value; } \\
\text { Light intensity value }\end{array}$ & NULL \\
\hline $\begin{array}{l}\text { plectrical } \\
\text { parameters }\end{array}$ & Common & $\begin{array}{l}\text { Voltage; Current; } \\
\text { Power factor; } \\
\text { Harmonic value }\end{array}$ & NULL \\
\hline $\begin{array}{l}\text { Person detection } \\
\text { CO2 } \\
\text { concentration }\end{array}$ & Common & Number of person & NULL \\
\hline $\begin{array}{l}\text { Air conditioning } \\
\text { terminal }\end{array}$ & Common & $\begin{array}{l}\text { Wind speed; } \\
\text { Setting } \\
\text { Working mode; } \\
\text { State }\end{array}$ & NULL \\
\hline $\begin{array}{l}\text { Abnormal } \\
\text { alarm }\end{array}$ & Common & State & NULL \\
\hline
\end{tabular}




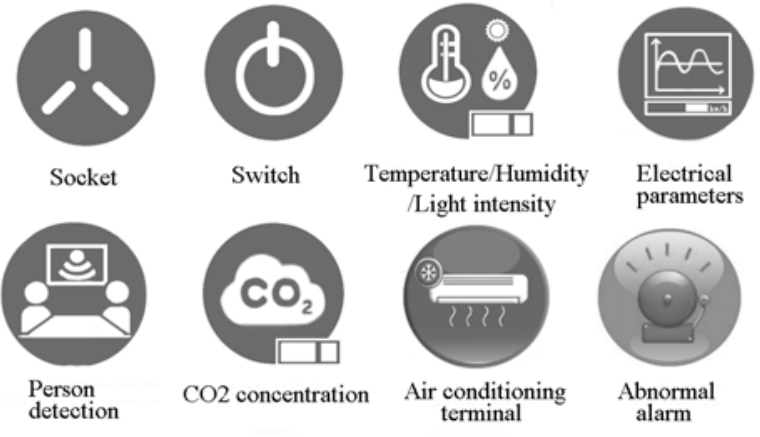

FIGURE III. TYPICAL INTELLIGENT DEVICE MODEL LOGO

The logo of the intelligent device is divided into two kinds: static and dynamic. The static device model logo only displays fixed information, which does not change the appearance of logo, while the dynamic device model logo changes according to the changes of the operation data. The dynamic device model logo is implemented by the way of image superposition, taking the temperature/humidity/light intensity model logo as an example, in which the temperature part is composed of two parts, as shown in Figure 4:

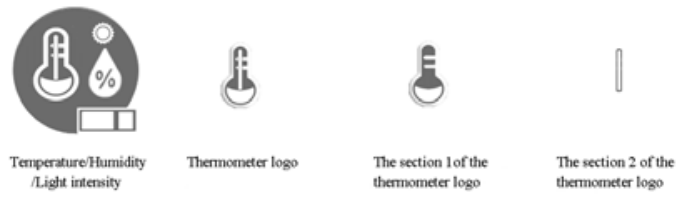

FIGURE IV. THE LOGO COMPOSITION OF TEMPERATURE PART

The section2's height property of the temperature logo is bound to the real temperature sensor's real-time data. When the system is running, the height of the section 2 of the themometer logo will follows the temperature value supported by the data center, so as to realize the dynamic effect of the logo.

\section{E. Design of the Data Processing Algorithm Model Library}

The disturbance of uncertain factors in the environment, signal processing difficulties in the hardware circuit of the electrical equipment and the defect and weakness of the hardware itself in the sensor measurement link will bring about the inaccuracy of the measurement data [5]. In this paper, the data processing algorithm model is used to solve the problem,which includes the digital filtering algorithm for overcoming large pulse interference (amplitude-limiting filtering algorithm, median filter algorithm) and the average filtering method for suppressing small amplitude high frequency noise (average filter algorithm, moving-weightedaverage algorithm)and so on. The data processing algorithm model is designed and implemented based on the objectoriented method, and applied between the real-time database and the intelligent device model.For example: it can eliminate the sharp pulse interference in the temperature measurement data by the amplitude-limiting filtering algorithm model, and prevent the false action of the air air-conditioner.The design of the typical data processing algorithm model is shown in Table2, the corresponding logo is shown in Figure 5.
TABLE II. TYPICAL DATA PROCESSING ALGORITHM MODEL

\begin{tabular}{|l|l|l|l|}
\hline \multicolumn{1}{|c|}{ Type } & $\begin{array}{l}\text { Num } \\
\text { ber } \\
\text { of } \\
\text { Input }\end{array}$ & $\begin{array}{c}\text { Numb } \\
\text { er of } \\
\text { Outpu } \\
\text { t }\end{array}$ & \multicolumn{1}{|c|}{ sarameter } \\
\hline $\begin{array}{l}\text { Average filter } \\
\text { algorithm }\end{array}$ & $\mathrm{N}$ & 1 & $\begin{array}{l}\text { Each input } \\
\text { weight } \\
\text { value }\end{array}$ \\
\hline $\begin{array}{l}\text { Amplitude- } \\
\text { limiting filter } \\
\text { algorithm }\end{array}$ & 1 & 1 & $\begin{array}{l}\text { Amplitude } \\
\text { value }\end{array}$ \\
\hline
\end{tabular}

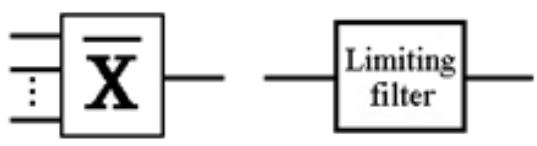

FIGURE V. TYPICAL DATA PROCESSING ALGORITHM MODEL LOGO

\section{TESTING AND APPLICATION}

The monitoring software is developed via a similar process of "piling blocks" based on the actual demand by using the models and components, users complete the construction of the monitoring software through a similar process of "piling blocks". There are only one configuration module and data center in one configuration software, while the running component can be one or more, so as to achieve one to one or one to many interaction modes between the data center and the running component. Taking a personal office of the passive laboratory building of Shandong Jianzhu University as an example, the process and contents of the configuration development of monitoring software are as follows:

The configuration component interacts with the data center to obtain the information of the intelligent device, including ID, name and type.The corresponding intelligent device models and the related data processing algorithm models are loaded to the monitoring interface designer automatically.

Using the monitoring interface designer, multiple room monitoring interface are created as the Basic management units. Using the actual room picture as a background, adding intelligent device models and data processing algorithm models to each room monitoring interface through drag and drop, and draging intelligent device models to the position where is corresponding with the actual position of intelligent devices,so as to implement the construction of each room monitoring interface rapidly.

Using parameter configuration tool to set up the relationship between the intelligent device models, data processing algorithm models, and real-time database.

Using the control logic editor to set the logic relationship between the parameters of each room's intelligent device model, such as the judgment condition, timer, etc.

Using the Engineering file output tool to save the entire pro ject as a data file in a specific format. 
Using the running component to load the data file, realizing the intelligent device real-time monitoring and controlling through the interaction with the data center.

In particular, when the monitoring software needs to be reconstructed, the intelligent device model corresponding to the type or number changed of intelligent devices in the space unit will be reloaded to the monitoring interface designer. Only need to complete the configuration of those intelligent device models, users can realize the reconstruction of the monitoring software. Figure 6 is the monitoring interface for a room in the configuration state, Figure 7 is the monitoring interface of a room in the running state, and Figure 8 is the logical con between each model in the room's monitoring interface and the real-time database.

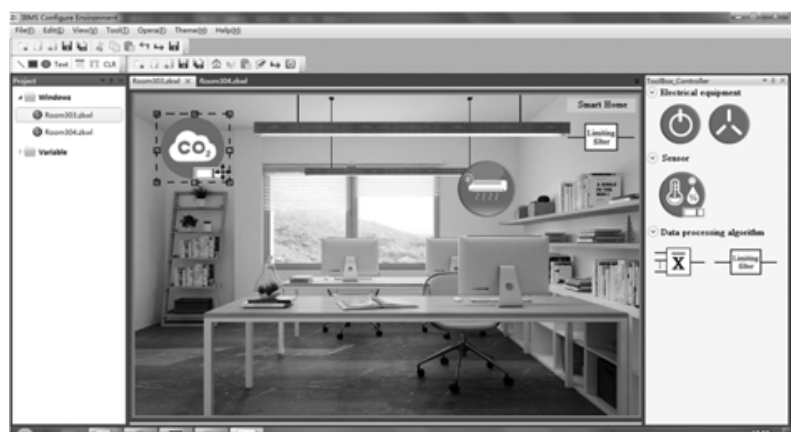

FIGURE VI. THE MONITORING INTERFACE FOR A ROOM IN THE CONFIGURATION STATE

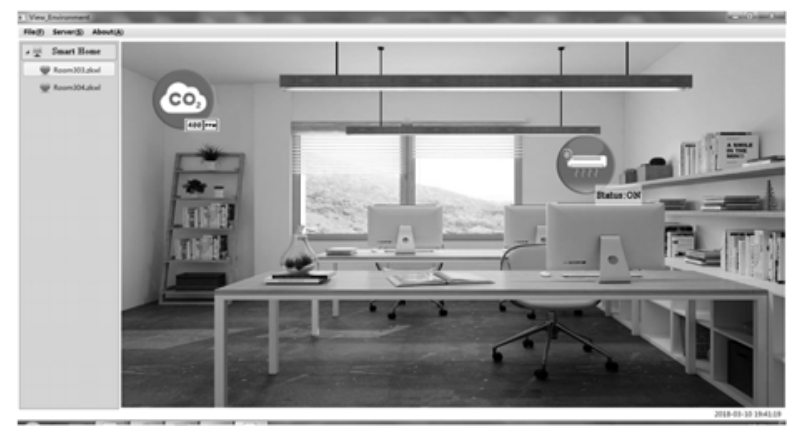

FIGURE VII. THE MONITORING INTERFACE OF A ROOM IN THE RUNNING STATE

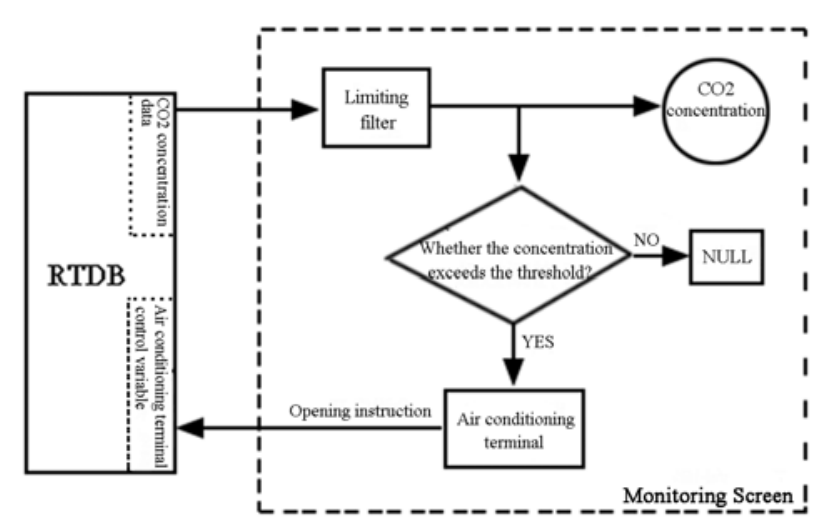

FIGURE VIII. THE LOGICAL CONNECTION BETWEEN EACH MODEL IN THE ROOM'S MONITORING INTERFACE AND THE REALTIME DATABASE.

\section{CONCLUSION}

A configuration software for the wireless ad hoc network monitoring system in intelligent building is designed and realized in this paper, provides the development-running environment, the intelligent equipment model, the data processing algorithm model, and encapsulates the custom TCP/IP communication protocol in the component form. Through configuration and customization, the software can be flexibly constructed by using the components of intelligent device model library, data processing algorithm model library and so on, so as to achieve the purpose of using space unit as the basic management unit, and realize the coordinated operation of intelligent devices that serving the same space. Compared with the traditional configuration software, the software has the advantages of flexibility and industry characteristic, and thus is worth popularizing in the field of intelligent building.

\section{REFERENCES}

[1] Dinh N T, Kim Y. RESTful Architecture of Wireless Sensor Network for Building Management System[J]. Ksii Transactions on Internet \& Information Systems, 2012, 6(1):46-63. .

[2] Shen B, Zhang G, Wang S, et al. The development of management system for Building Equipment Internet of Things[C]// IEEE, International Conference on Communication Software and Networks. IEEE, 2011:423-427.

[3] Yao Y, Yao Y, Zhu W. Fuzzy Control Algorithm in Configuration Software's Application and Research[C]// IEEE International Conference on Software Engineering and Service Sciences. 2010:292 - 294.

[4] Qiao Y, Zhang G, Ming W, et al. Design of the room controller in the Internet of Things for building electrical equipments[C]// Industrial Electronics and Applications. IEEE, 2011:834-838.

[5] Pop S, Dan P, Ciascai I. Adaptive algorithm for error correction from sensor measurement[C]// International Spring Seminar on Electronics Technology. IEEE, 2009:373-378. 